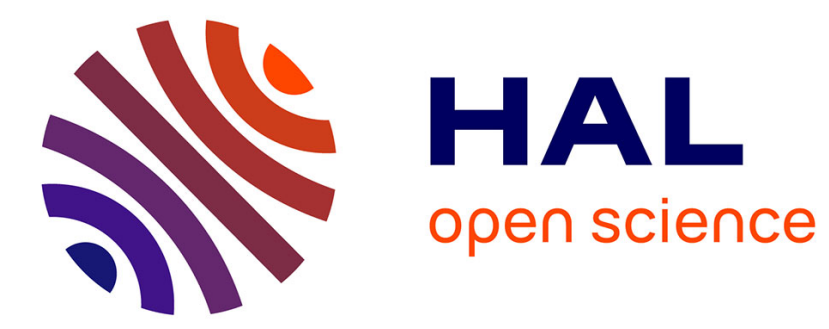

\title{
Le déchet, matière à réflexion
}

Cyril Harpet

\section{- To cite this version:}

Cyril Harpet. Le déchet, matière à réflexion. Environnement, Ingénierie \& Développement, 1996, ํ1

- 1er Trimestre 1996, pp.11-12. 10.4267/dechets-sciences-techniques.552 . hal-03155555

\section{HAL Id: hal-03155555 \\ https://hal.science/hal-03155555}

Submitted on 1 Mar 2021

HAL is a multi-disciplinary open access archive for the deposit and dissemination of scientific research documents, whether they are published or not. The documents may come from teaching and research institutions in France or abroad, or from public or private research centers.
L'archive ouverte pluridisciplinaire HAL, est destinée au dépôt et à la diffusion de documents scientifiques de niveau recherche, publiés ou non, émanant des établissements d'enseignement et de recherche français ou étrangers, des laboratoires publics ou privés. 


\section{LE DÉCHET, MATIËRE À RÉFLEXION}

Cyril Harpet*

Doctorant Lyon III, option Epistémologie, étude des systèmes

En quoi le déchet peut-il intéresser un philosophe ? Ce dernier jusque-là avait sans doute des préoccupations d'un tout autre ordre, des questions en apparence plus fondamentales à soulever. Qu'apporterait-il en ce domaine aussi éloigné des thèmes propres à sa démarche ? Mais c'est là supposer encore l'appartenance du philosophe à une sorte de "classe " ou de caste qui ne vit et ne se développe qu'à travers des débats périmés, ne se prêterait qu'à un certain type de discours tantôt moralisateur, tantôt abstrait au point d'être ésotérique. Or il n'en est rien : rappelons tout d'abord qu'aucune question ne saurait lui être étrangère ou vaine du moment qu'elle éveille la conscience de chacun et qu'elle est rattachée à une problématique. Ajoutons qu'ensuite le domaine de la matière ne saurait être investi et interrogé par les seuls tenants et spécialistes de la technologie ou de l'industrie. Car en effet, traiter de la matière suppose une implication humaine qui en dépasse largement les qualités et propriétés strictement techniques. Autrement dit un champ de représentations prédispose l'homme à traiter la matière selon des principes théoriques et pratiques en évolution constante. La matière n'est point définie et traitée comme telle puisqu'elle exige un rapport doté de qualités sensorielles, de schémas conceptuels spécifiques.

Le philosophe est trop souvent décrit selon un profil qui le met hors du jeu des préoccupations d'ordre matériel. Or celui-ci occupe progressivement une place prépondérante au sein de notre civilisation, ce qui ne saurait admettre une ignorance ou un refus d'en interroger les modalités de traitement. Quand nous parlons de traitement, nous entendons aussi bien celui cognitif, que l'activité scientifique en général suppose, que celui technologique auquel est consacré tout un pan de l'activité industrielle contemporaine.

Si la question « qu'est-ce que la matière ? » a pu être posée régulièrement à travers l'histoire de la pensée humaine, il en est une qui lui est apparentée et dont nous n'avons constaté que trop peu un approfondissement : « qu'est-ce qu'un déchet? ». En effet il semble qu'une frontière épistémologique ait été tracée entre ce qui relève du domaine de la matière au sens noble du terme et celui des matières ingrates, inutiles ou déchues. Cette frontière, parfois une véritable muraille, est à franchir : elle s'étend vers le haut, selon une échelle des qualités au sommet de laquelle règnent les chef d'œuvres, au bas de laquelle s'abîment les restes, les rebuts. La tâche est d'autant plus ardue que cet obstacle épistémologique aura été longuement constitué et élevé au cours de l'histoire de la pensée du fait des représentations et des postulats qui ont présidé à une totale disqualification de ce qui est « ignoble ». Un champ « maudit » a été ainsi délimité dans l'espace d'activité intellectuelle de l'homme de même qu'il a été circonscrit dans l'espace de sociabilité. Les partages opérés touchent divers niveaux de la réalité humaine : nous en distinguons quatre :

- le champ maudit de la corporéité : l'espace du corps est lui-même connoté en ses régions puisque certaines parties restent soumises à des emplois restrictifs, à des prohibitions, à des pratiques de soins particuliers (en général les « orifices »);

- le champ maudit du langage : le langage comprend des unités linguistiques qui portent le sceau de la corruption ;

- le champ maudit de l'espace social et culturel : l'espace est découpé selon des axes de partage et réparti en secteurs signifiés, puis aménagé et occupé dans le respect de ces règles de distribution et de ces significations (l'aménagement des milieux urbain et rural) ;

- le champ maudit de la nature : les éléments vivants ou non composant le domaine de la nature sont rattachés à des catégories signifiées (le consommable - le non consommable, l'utile et le nuisible, le diurne - le nocturne, la proie - les animaux de proie...).

Le philosophe, tout comme le scientifique, n'a à craindre aucun de ces territoires incongrus, prétendument « dangereux », et de ce fait interdits ; bien au contraire, l'investigation de ces " marges » ou de ces antres lui revient : il doit être l'agent d'une pensée généreuse et courageuse. II ne peut se résoudre à une attitude timorée et frileuse au bord des marges : il doit explorer les limites et l'au-delà de ces limites. II ne peut se contenter d'en suivre les contours, il lui faut avancer le pas, outrepasser les limites. Aussi, c'est pourquoi, il nous a paru déterminant de s'interroger non plus sur la matière, celle " polie », épurée et définie en fonction d'une finalité productive ou productiviste. Nous 
préférons aborder celle « sauvage », abandonnée, déchue, désuète, a priori inutile. Ce n'est plus la matière prise dans un processus complexe de transformation et de normalisation déjà bien entamé qui nous intéresse, mais celle encore disqualifiée, timidement traitée même s'il nous faut constater son entrée marquante dans le processus de traitement et de revalorisation. Car le déchet est encore soumis à un procès depuis longtemps entamé à son encontre dont peu à peu il est permis de penser que la science et les technologies l'en extraient.

Les thèmes classiques de la philosophie et les objets de son intarissable questionnement portent sur des valeurs (la justice, la vérité, le pardon, la politesse...), sur des concepts (la notion de structure, le système, le mouvement...) ou sur des disciplines et des domaines de la connaissance (l'histoire, la science, la philosophie...). Rarement se sont trouvées posées des questions concernant une notion qui importe autant par sa matérialité que par le chapelet conceptuel qu'elle égrène. Certes des illustrations par des faits, des événements concrets tirés d'une expérience personnelle ou historique viennent conférer une assise réelle à l'argumentation en vigueur, mais c'est bien souvent par des rapprochements nécessaires à la démonstration et non matériellement compris dans la notion. II en va tout autrement du déchet : à lui seul, ce terme renvoie spontanément à la dimension matérielle qu'il définit en même temps qu'il définit une catégorie, un paradigme qui dépasse largement cette première dimension. La franche polarité matérielle à laquelle il nous convie d'emblée ne saurait nous faire omettre les diverses connotations et prolongements conceptuels que le terme de déchet contient.

\section{LE DÉCHET TRAITÉ ET MALTRAITÉ}

Ainsi nous sommes philosophes ! Et nous préparons actuellement une thèse d'épistémologie sur le déchet! Que peut donc apporter un tel énergumène en un domaine a priori aussi éloigné de ses préoccupations classiques?

Tout philosophe est en droit de s'interroger sur les diverses activités de ses contemporains, ce qui ne l'écarte point d'y participer et de s'y mêler activement. Mais il est de son devoir et de sa formation d'aller au-delà des apparences sinon plus loin que ce qui lui est présenté, nous l'avons dit. Et le déchet s'est vu jusqu'ici selon nous l'objet de spécialistes de son traitement technique, de ses modes de gestion et résorption : mais ce qui est à évacuer, transformer ou à enfouir attise la curiosité de bien des hommes pour une investigation des recoins, des espaces malfamés, des lieux « détritiques ». Le philosophe est pour nous cet inlassable curieux qui ne se contente guère de ce qui est présenté comme d'emblée devant être soustrait à son regard, à son analyse, à sa réflexion. Ainsi évacuez, transformez, enterrez, nous saurons bien trouver des détours afin de savoir ce qui se prépare hors de nos sens. Le travail du philosophe consiste non plus à contester un pouvoir établi, qui s'affiche et s'affirme comme tel, mais plutôt de nos jours à inspecter les marges, les sous-bassements, les arrière-cours, les arrière- pensées au besoin : il est l'investigateur des lieux les plus reculés pour y exercer justement sa faculté de recul. Car il en est bien ainsi : ce qui est soustrait à notre sensibilité, à nos sens l'est d'autant plus qu'il y a risque d'effroi, de stupeur, d'angoisse de la contagion. Et que dire donc des montagnes d'ordures qui s'élèvent hors de nos cités? Elles n'incitent guère à les gravir tellement elles laissent échapper des effluves pestilentielles, tellement elles deviennent les foyers des bêtes de l'immonde qui occupent une fantasmagorie collective (les rats en particulier). Que viendrait faire un philosophe en ces lieux maudits? Lui si pétri d'idéaux et d'idées nobles, que viendrait-il traîner ses guêtres là où s'étalent les marques et les aveux de la finitude de notre civilisation? A moins que justement n'en soit là le commencement ! C'est la prendre à revers cette fois, que de longer puis de franchir ses frontières en passant par ce qu'elle exècre et évacue. Lacan déjà l'écrivait : « La civilisation, c'est le déchet : cloaca maxima ». Le grand et vaste cloaque.

\section{* Cyril Harpet}

Doctorant Lyon III, option Epistémologie, étude des systèmes - 85, Grande rue de la Croix Rousse - 69004 Lyon 